\title{
Higher Order Corrections to Spin Correlations in Top Quark Pair Production at the LHC
}

\author{
Arnd Behring \\ Institut für Theoretische Teilchenphysik und Kosmologie, RWTH Aachen University, D-52056 Aachen, Germany \\ and Institute for Theoretical Particle Physics, KIT, D-76128 Karlsruhe, Germany \\ Michał Czakon \\ Institut für Theoretische Teilchenphysik und Kosmologie, RWTH Aachen University, D-52056 Aachen, Germany \\ Alexander Mitov and Rene Poncelet \\ Cavendish Laboratory, University of Cambridge, Cambridge CB3 OHE, United Kingdom \\ Andrew S. Papanastasiou \\ Cavendish Laboratory, University of Cambridge, Cambridge CB3 OHE, United Kingdom \\ and MRC Institute of Genetics and Molecular Medicine, University of Edinburgh, \\ Crewe Road, Edinburgh EH4 2XU, United Kingdom
}

(Received 5 February 2019; published 21 August 2019)

\begin{abstract}
We calculate, for the first time, the next-to-next-to-leading order (NNLO) QCD corrections to spin correlations in top quark pair production at the LHC. The NNLO corrections play an important role in the description of the corresponding differential distributions. We observe that the standard model calculation describes the available $\Delta \phi_{\ell \ell}$ data in the fiducial region but does not agree with the $\Delta \phi_{\ell \ell}$ measurement extrapolated to full phase space. Most likely this discrepancy is due to the difference in precision between existing event generators and NNLO calculations for dilepton top-pair final states.
\end{abstract}

DOI: 10.1103/PhysRevLett.123.082001

Introduction.-Within the standard model (SM) of particle physics, individual top quarks produced in proton-(anti)proton collisions are not polarized. The spins of two pair-produced top quarks are, however, correlated to each other. It is possible [1] to study directly such spin correlations between top quarks since, due to the very rapid decay of the top quark, its spin is passed to its decay products almost free of nonperturbative effects [2]. This implies that top quark spin correlations are calculable.

The study of top quark spin correlations has a long history. Spin correlations have been long recognized as a powerful tool for probing the nature of the quark sector in the SM [3-14] as well as Higgs and/or beyond the SM (BSM) physics [15-19]. Indeed, a generic BSM contribution to top production will alter the top-pair production spin density matrix. An important example is the case of a light spin zero top quark supersymmetric partner, the stop, decaying to top quarks $[16,18]$. Seeking deviations between SM predictions and LHC measurements of top quark spin correlations represents a powerful, model-independent

Published by the American Physical Society under the terms of the Creative Commons Attribution 4.0 International license. Further distribution of this work must maintain attribution to the author(s) and the published article's title, journal citation, and DOI. Funded by SCOAP ${ }^{3}$. search strategy for possible BSM physics coupled to the top quark sector.

Very recently, the ATLAS Collaboration published [20] a very precise measurement of spin correlations in top quark pair production at the LHC (earlier LHC and Tevatron measurements include Refs. [21-25]). A deviation of about $3.2 \sigma$ with respect to the SM has been observed. This is by far the biggest deviation from the SM observed in the top quark sector at the LHC to date. Given the potential significance of such a discrepancy, in this Letter we calculate for the first time the complete set of NNLO QCD corrections to top quark pair production and decay. Our calculation uses the narrow width approximation. It allows us to qualitatively increase the level of precision of SM predictions for realistic top quark final states, thus making the comparison with the ATLAS data [20] much more predictive.

Generally, top quark spin correlations can be assessed following two strategies. The first strategy, which we call direct, reconstructs the top-pair spin density matrix and is based on kinematic distributions computed in specially designed frames of reference; see Refs. [8,9] for details.

The second strategy, which we call indirect, utilizes differential distributions defined in the laboratory frame. These distributions are best suited for experimental study but they tend to be only partly sensitive to spin correlations. In order to maximize the extracted information about spin 
correlations, the use of a likelihood function was advocated for in Ref. [10]. Clearly, a prerequisite for extracting spin correlations from laboratory frame distributions is good control over theory predictions.

In this Letter we use the indirect approach to spin correlations and study the following differential distributions: the angular difference $\Delta \phi_{\ell \ell}$ between the two leptons in the transverse plane and the rapidity difference $\left|\Delta \eta_{\ell \ell}\right|$ between the two leptons. Both observables are sensitive to spin correlations and can be measured with high precision since the top quarks need not be reconstructed.

Our main goal in this Letter is to establish whether higher order corrections can account for the $3.2 \sigma$ discrepancy in the $\Delta \phi_{\ell \ell}$ distribution reported in Ref. [20]. Our finding is in the affirmative. In hindsight, this should not come as a complete surprise given the important role higher order QCD corrections play in the $t \bar{t}$ forward-backward asymmetry [26] and in taming the so-called top $p_{T}$ discrepancy [27]. We caution, however, that the interpretation of higher order corrections is not completely straightforward since it uncovers possible subtleties in the modeling of realistic top quark final states at hadron colliders. We explain all of this in detail in the results section.

Details about the calculation.-The calculations performed in this Letter are at next-to-next-to-leading order (NNLO) in QCD. This means that NNLO QCD corrections to both top-pair production and top quark decay are included. This is the first time top quark pair production and decay has been consistently computed in NNLO QCD.

As in Ref. [28], where top-pair production was included in approximate NNLO, the present calculation is performed within the narrow width approximation for both the top quark and the $W$ boson. This approximation is known to work well [29] for distributions that are away from kinematic boundaries, which is the case considered in this Letter. Below we also compare our calculation with a more recent NLO study [30]. We recall that this approximation has been used in the existing NNLO QCD calculations for single top production including top decay $[31,32]$.

We consistently truncate the production $\times$ decay differential cross section through NNLO in QCD:

$$
\begin{aligned}
d \sigma^{\mathrm{LO}}= & d \sigma^{\mathrm{LO} \times \mathrm{LO}}, \\
d \sigma^{\mathrm{NLO}}= & d \sigma^{\mathrm{NLO} \times \mathrm{LO}}+d \sigma^{\mathrm{LO} \times \mathrm{NLO}}-\frac{2 \Gamma_{t}^{(1)}}{\Gamma_{t}^{(0)}} d \sigma^{\mathrm{LO}}, \\
d \sigma^{\mathrm{NNLO}}= & d \sigma^{\mathrm{NNLO} \times \mathrm{LO}}+d \sigma^{\mathrm{LO} \times \mathrm{NNLO}}+d \sigma^{\mathrm{NLO} \times \mathrm{NLO}} \\
& -\frac{2 \Gamma_{t}^{(1)}}{\Gamma_{t}^{(0)}} d \sigma^{\mathrm{NLO}}-\frac{\left(\Gamma_{t}^{(1)}\right)^{2}+2 \Gamma_{t}^{(0)} \Gamma_{t}^{(2)}}{\left(\Gamma_{t}^{(0)}\right)^{2}} d \sigma^{\mathrm{LO}} .
\end{aligned}
$$

As the above equations imply, the top quark decay width has also been expanded in powers of $\alpha_{S}$ [as in Eq. (2)]. The contribution containing NLO corrections to the two decays is included in $d \sigma^{\mathrm{LO} \times \mathrm{NNLO}}$.
The $d \sigma^{\mathrm{NLO}}$ correction has been known for some time [33-35]. These results were extended in Ref. [36] to include approximate NNLO results in production, while Ref. [28] combined the approximate NNLO correction in production with the complete NNLO correction in decay.

Our calculation uses the STRIPPER framework [37-39] for NNLO calculations in QCD. The only exception is the calculation of the $d \sigma^{\mathrm{NLO} \times \mathrm{NLO}}$ contribution, where, purely for convenience, the decay correction is computed with the help of Catani-Seymour dipoles [40,41] as implemented in Ref. [42].

We modify the existing calculations of differential toppair production $[27,43,44]$ in such a way that the information about the helicities of the top quarks is retained. For the double real correction at NNLO, this requires the use of tree-level helicity amplitudes. The real-virtual corrections require the calculation of the one-loop five-point helicity amplitudes for the processes $q \bar{q} \rightarrow t \bar{t} g, q g \rightarrow t \bar{t} q$, and $g g \rightarrow t \bar{t} g$. To that end we have used a private version [45] of the OPENLOOPS2 code [46] which employs the stability and speed improvements of Ref. [47]. We have checked that the result agrees with a modified version of a private code by S. Dittmaier used previously in the calculation of spin-averaged top production. The two-loop amplitudes $q \bar{q} \rightarrow t \bar{t}$ and $g g \rightarrow t \bar{t}$ have been computed in Ref. [48] using spin projections and the methods used for the derivation of the spin-averaged amplitudes [49].

We have computed independently the NNLO QCD correction to top decay. The two-loop helicity amplitude $t \rightarrow b W(\rightarrow \ell \nu)$ is known analytically [50-52]. The oneloop helicity amplitude for $t \rightarrow b g W(\rightarrow \ell \nu)$ has been computed in analytical form in Ref. [53] and has been checked numerically against the OPENLOOPS and GoSAM [54] libraries. Alternatively, we have implemented the results of Ref. [42] and find agreement between the two. We have checked that the assembled fully differential spinaveraged top quark decay width agrees within numerical uncertainties with the program NNTOPDEC [55] as well as with Ref. [56] (where such a comparison was possible).

For all terms in Eq. (1) but $d \sigma^{\mathrm{NNLO} \times \mathrm{LO}}$, we have checked that they agree with Ref. [28].

In the calculation we work in the $G_{\mu}$ scheme and use the following set of numerical inputs:

$$
\begin{aligned}
m_{t} & =172.5 \mathrm{GeV}, \\
m_{W} & =80.385 \mathrm{GeV}, \\
m_{Z} & =91.1876 \mathrm{GeV} \\
\Gamma_{W} & =2.0928 \mathrm{GeV}, \\
\Gamma_{t} & =\left(1.48063-1.18 \alpha_{S}-2.65 \alpha_{S}^{2}\right) \mathrm{GeV}, \\
G_{F} & =1.166379 \times 10^{-5} \mathrm{GeV}^{-2}
\end{aligned}
$$

The top quark width is specified as an $\alpha_{S}$ expansion through NNLO in QCD [55-57], as needed in Eq. (1). 
It is treated as a fixed parameter throughout this Letter, and its value in Eq. (2) corresponds to a fixed scale $\mu=m_{t}$.

In this Letter we take the $b$ quark to be massless and renormalize with $n_{F}=5$ active flavors. The top quark is renormalized on shell; i.e., we use the top quark pole mass. Its value $m_{t}=172.5 \mathrm{GeV}$ is lower than the world average. It is chosen such that it agrees with the value used by the ATLAS Collaboration. This way our predictions can be directly compared with Ref. [20].

We use the NNPDF3.1 [58] family of parton distributions but have also checked the CT14 set [59]. The default renormalization and factorization scales are chosen dynamically as proposed in Ref. [44]:

$\mu_{F, R}=\frac{H_{T}}{4}, \quad H_{T}=\sqrt{m_{t}^{2}+p_{T, t}^{2}}+\sqrt{m_{t}^{2}+p_{T, \bar{t}}^{2}}$.

In the evaluation of the scales equation (3) we have used the true top momenta, not the reconstructed ones.

Although our setup allows us to output FASTNLO tables $[60,61]$ in this first NNLO calculation with top decay, we have chosen for simplicity not to do so. We plan to use this capability in future calculations as with our results [62] of stable top quark production. All results derived in this Letter, together with some extra plots, are available for download from Ref. [63].

Results.-In this Letter we calculate two differential distributions-namely, the two leptons' angular difference in the transverse plane $\Delta \phi_{\ell \ell}$ and their rapidity difference $\left|\Delta \eta_{\ell \ell}\right|$.

We have two selection criteria for each distribution. The first one, called inclusive, does not assume any selection cuts. The second one, called fiducial, is based on the ATLAS selection cuts [20]: an electron and a muon of opposite electric charge with $p_{T}>27(25) \mathrm{GeV}$ for the harder (softer) lepton and $|\eta|<2.5$. In addition, we require at least two jets (at least one of which is a $b$-flavored jet) with $p_{T}>25 \mathrm{GeV}$ and $|\eta|<2.5$. All jets are defined with the anti- $k_{T}$ algorithm [64] with $R=0.4$.

The normalized fiducial and inclusive $\Delta \phi_{\ell \ell}$ and $\left|\Delta \eta_{\ell \ell}\right|$ distributions are shown in Figs. 1 and 3, respectively. Each curve is normalized with respect to the corresponding visible cross section; i.e., the integral under it equals unity. The $\Delta \phi_{\ell \ell}$ distribution is compared with the published ATLAS data [20]; the $\left|\Delta \eta_{\ell \ell}\right|$ one is not since the corresponding data have not been published yet.

A number of observations can be made from Fig. 1. The most interesting feature is the different behavior of the NNLO/NLO $\Delta \phi_{\ell \ell} K$ factor between the fiducial and inclusive cases. With respect to the inclusive case, in the fiducial case the $K$ factor is much larger, the NNLO distribution is in good agreement with the data, and the scale uncertainty is much larger. Notably, the NNLO inclusive prediction does not agree well with the data.

Since both the fiducial and inclusive data originate from the same measurement, it is not a priori clear why the
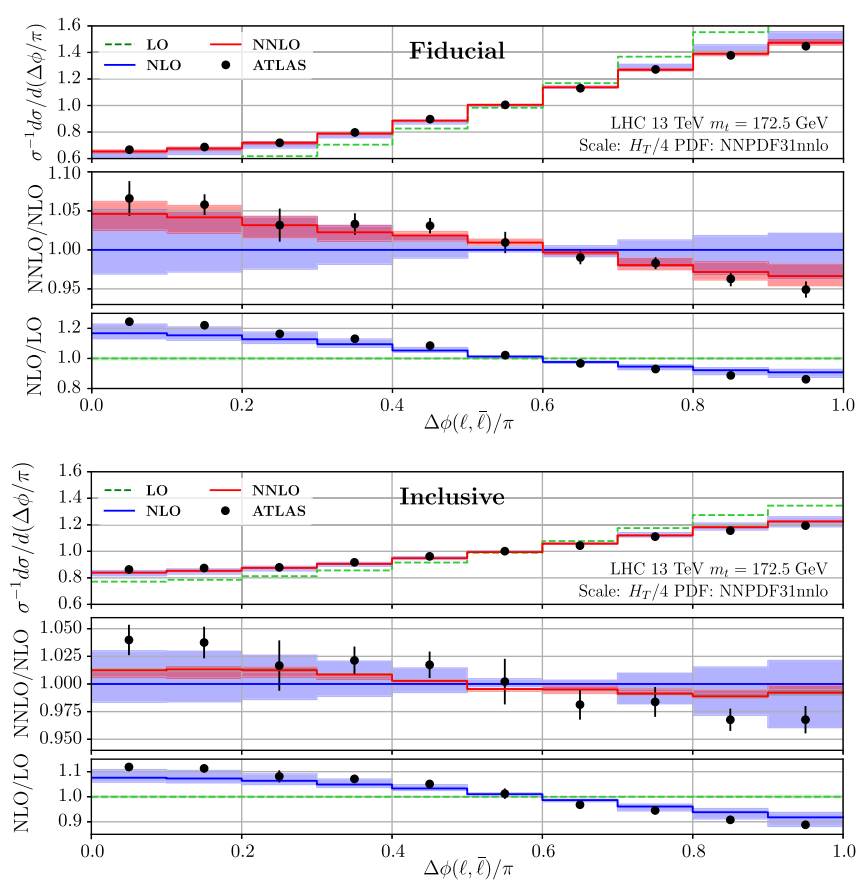

FIG. 1. NNLO QCD predictions for the (top panels) fiducial and (bottom panels) inclusive selections of the normalized $\Delta \phi_{\ell \ell}$ distribution versus the ATLAS data [20]. Uncertainty bands are from seven-point scale variation.

NNLO calculation would agree with only one of them. In our view the most plausible explanation for this discrepancy lies in the extrapolation of the fiducial measurement to the full phase space.

Such a conclusion should not come as a complete surprise since the extrapolation to full phase space is performed with event generators that have accuracy different than the one in this Letter. In fact an early indication about the importance of higher order corrections in top quark production came from the long-standing top quark $p_{T}$ discrepancy-namely, that NLO-accurate event generators do not model well the LHC top quark $p_{T}$ distribution (see, e.g., Refs. [65-67]), while the NNLO QCD correction significantly improves the agreement with data [27].

Anatomy of higher order corrections to $\Delta \phi_{\ell \ell}$ : - In the following we offer a detailed analysis quantifying a number of possible contributions to this observable. We show that they are too small to affect the behavior of this observable in the SM.

(i) Is the NNLO correction large?: - NLO analyses [20] indicate that higher order effects are likely not going to bridge the $3.2 \sigma$ discrepancy with the ATLAS $\Delta \phi_{\ell \ell}$ data. Yet we see that the NNLO QCD prediction agrees well with the data in the fiducial region. From this one cannot directly conclude that the NNLO correction is unusually large. The reason is that our NNLO prediction uses scales different from the ones in most event generators.

For our preferred choice of scales we find that the fiducial NNLO/NLO $K$ factor is no larger than $5 \%$. This is 
a perfectly reasonable NNLO correction which, moreover, is consistent with the NLO scale uncertainty band. The $\mathrm{NLO} / \mathrm{LO} K$ factor is larger by a factor of about 3. In the inclusive case one observes smaller $K$ factors and less scale variation, which is reasonable to expect since the observable is more inclusive. We note that in both cases the smallness of the LO uncertainty band is due to a cancellation between the normalization factor and is not representative of the true uncertainty in the differential distribution.

We conclude that the behavior of $\Delta \phi_{\ell \ell}$ is consistent with good perturbative convergence. The NNLO correction plays an important role: in the fiducial case it reduces the scale uncertainty by more than a factor of 2 and modifies the slope of the theory prediction in a direction that improves the agreement with data.

(ii) Choice of scales: - All calculations in this Letter are performed with three scales: the one in Eq. (3) as well as $\mu_{F, R}=m_{t}$ and $\mu_{F, R}=m_{t} / 2$. As can be seen in Fig. 2, the result with scale $m_{t} / 2$ behaves similarly to the one in Eq. (3) and is even closer to the data. On the other hand, the calculation with scale $m_{t}$ has a larger NNLO/NLO $K$ factor, and the agreement with data in the fiducial case is not as good as with the other two scales.

To understand this behavior, we recall that the scale $\mu_{F, R}=m_{t} / 2$ was found in Ref. [44] to lead to fast perturbative convergence for the total cross section. This behavior is similar to the default dynamic scale of Eq. (3). However, perturbative convergence with the canonical scale $\mu_{F, R}=m_{t}$ is slower. We conclude that the pattern of higher order corrections for the fiducial $\Delta \phi_{\ell \ell}$ distribution is in line with our previous findings for generic top quark differential distributions. We expect that the predictions based on the default dynamic scale as well as on the scale $\mu_{F, R}=m_{t} / 2$ will not have significant corrections beyond NNLO. By contrast, the scale $\mu_{F, R}=m_{t}$ may lead to non-negligible corrections beyond NNLO, which is the reason, we believe, that it does not describe the data as well.

(iii) Value of $m_{t}$ :-With the help of a NLO calculation, we have checked that the value of the top quark mass does not affect the $\Delta \phi_{\ell \ell}$ distribution in a significant way. This may be expected on purely dimensional grounds $\left(\Delta \phi_{\ell \ell}\right.$ is a dimensionless variable). Nevertheless, a dedicated analysis is warranted in light of the findings of Ref. [68], where it

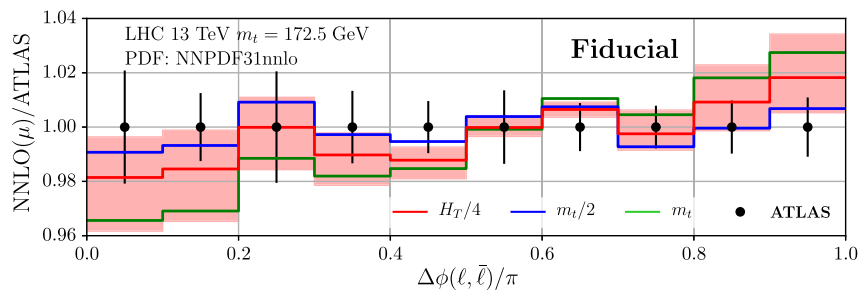

FIG. 2. Three NNLO QCD predictions utilizing different scales versus the ATLAS data [20]. The red band represents the sevenpoint scale variation for the default scale choice, Eq. (3). was found that the treatment of spin correlations in certain lepton distributions does have a substantial impact on the extracted top mass.

(iv) Parton distribution function (PDF) dependence: The effect on the normalized $\Delta \phi_{\ell \ell}$ distribution is at the level of $1 \%$ and thus is marginal. We have checked this by comparing two different PDF sets (NNPDF3.1 and CT14), including their PDF errors.

(v) Finite width and electroweak corrections: - We have performed a qualitative check of these effects at NLO using the results of Ref. [30]. While the setup for that reference is different from ours, the comparison indicates that the effects on the $\Delta \phi_{\ell \ell}$ distribution are small, perhaps of the order of $1 \%$. It will be very valuable to investigate such effects in detail in the future.

(vi) Top production versus top decay: -We find that radiative corrections to top quark decay have a small impact on the $\Delta \phi_{\ell \ell}$ distribution.

Observables other than inclusive $\Delta \phi_{\ell \ell}$ : -Following Refs. [8,20], we have also investigated the $\Delta \phi_{\ell \ell}$ distribution for several "slices" of the $t \bar{t}$ invariant mass $m_{t \bar{t}}$. Owing to space limitations, we present no results here (however, see Ref. [63]) but only remark that the NNLO corrections are small, in the sense that they are well within the NLO scale uncertainty band and have a much reduced scale variation relative to NLO. Interpreting such results is, however, subtle since our definition of $m_{t \bar{t}}$ is based on the true top momenta, unlike the experimental setup, where
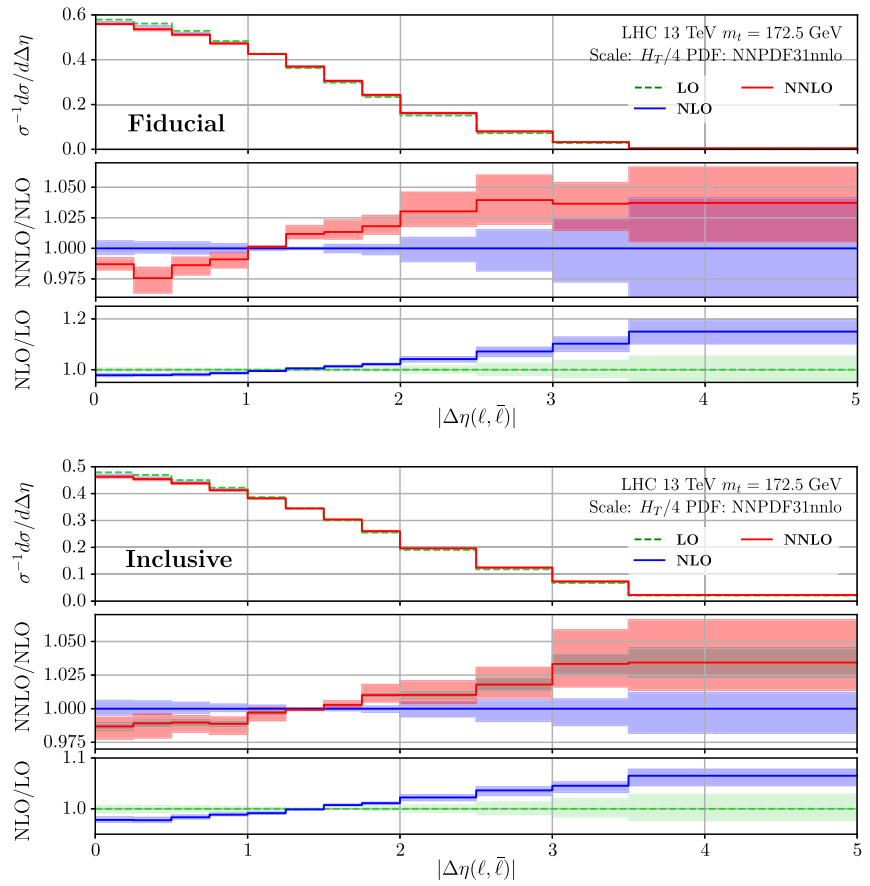

FIG. 3. NNLO QCD predictions for the (top panels) fiducial and (bottom panels) inclusive selections of the normalized $\left|\Delta \eta_{\ell \ell}\right|$ distribution. Uncertainty bands are from seven-point scale variation. 


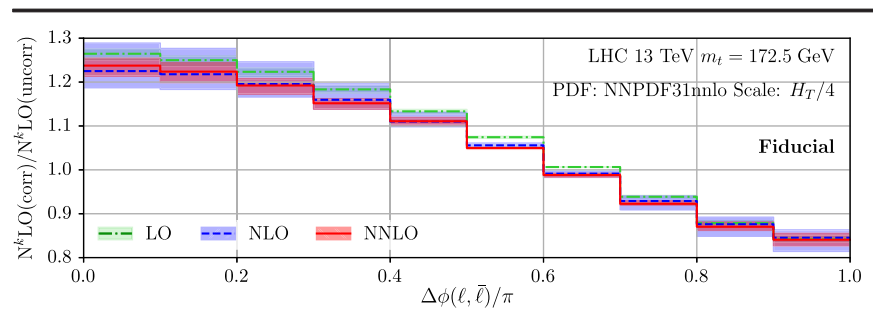

FIG. 4. Size of spin correlations in the fiducial $\Delta \phi_{\ell \ell}$ distribution at each order through NNLO in QCD.

the tops are reconstructed (see also Refs. $[8,10]$ for a discussion of this point).

Finally, in Fig. 3 we show the fiducial and inclusive predictions for the $\left|\Delta \eta_{\ell \ell}\right|$ distribution. Unlike the $\Delta \phi_{\ell \ell}$ distribution, the NNLO corrections are significant both in the fiducial and inclusive cases. It will be very interesting to compare these predictions with the data once they become available. An agreement of our NNLO prediction with future data is likely to validate our interpretation of higher order corrections in the $\Delta \phi_{\ell \ell}$ distribution discussed in the subsection pertaining to the anatomy of higher order corrections.

Quantifying spin correlations: - In Fig. 4 we show the magnitude of spin correlations in the $\Delta \phi_{\ell \ell}$ distribution through NNLO in QCD. To that end we take the ratio of the calculations with and without spin correlations at a given order. The former calculation is performed by taking spinaveraged top-production times spin-averaged top decay. We observe that spin correlations are large and change little at higher orders.

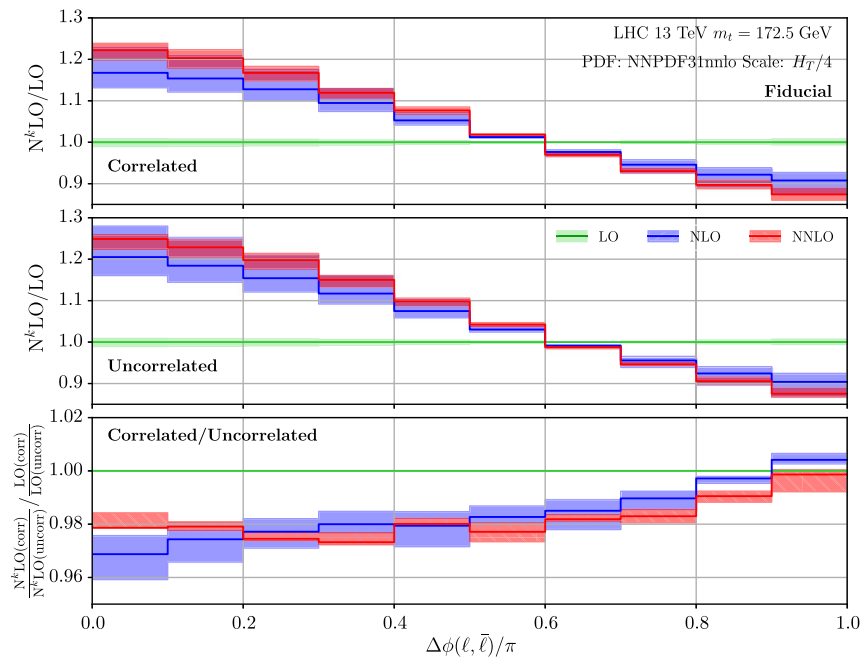

FIG. 5. Disentangling radiative corrections from spin correlations for the fiducial $\Delta \phi_{\ell \ell}$ distribution. Shown is the ratio $\mathrm{N}^{k} \mathrm{LO} / \mathrm{LO}$, for $k=0,1,2$, (top panel) for the spin-correlated calculation, (middle panel) for the calculation without spin correlation, and (bottom panel) for their ratio. The bands represent the spread of the ratios for each of the seven scale variations.
In order to disentangle the effect of kinematics from spin correlations, in Fig. 5 we show the ratios NLO/LO and NNLO/LO separately for the exact (top panel) and spinuncorrelated (middle panel) cases. We observe that all of these $K$ factors are significant in size and nearly identical to each other at a given perturbative order. This means that while higher order corrections are substantial they largely decouple from spin correlations. Indeed, the difference between the two NLO/LO and NNLO/LO bands is much smaller than their individual magnitudes. This can be seen more clearly in the bottom panel, where their ratio is taken.

Our analysis shows that the control of higher order corrections in the $\Delta \phi_{\ell \ell}$ distribution is essential for interpreting spin correlations with high precision. This is because in this observable spin correlations and kinematics are mixed in a very nontrivial way, and therefore a detailed analysis of spin correlations requires a good understanding of kinematic effects.

Conclusions. - In this Letter we compute, for the first time, the complete set of NNLO QCD corrections to toppair production and decay at hadron colliders. We work in the narrow width approximation for both the top quark and the $W$ boson. We utilize this calculation for the study of spin correlations in top-pair production in the dilepton channel.

Our calculation shows that NNLO QCD corrections to realistic dilepton top quark pair final states play an important role: they increase the SM prediction, significantly decrease the dominant scale uncertainty, and improve the agreement with data.

Using the scales advocated for previously in the context of stable top production, we find that NNLO QCD agrees with the recent $13 \mathrm{TeV}$ ATLAS data, thus alleviating, or perhaps removing altogether, the earlier reported $3.2 \sigma$ discrepancy with respect to the SM.

An important finding of this Letter is that data extrapolation to full phase space with existing event generators seems not to be compatible with the direct NNLO QCD calculation. We believe that, thanks to the very high precision of both theory predictions and experimental measurements, we begin to see clear evidence that top quark measurements begin to resolve and constrain such delicate modeling effects.

We thank the authors of the OpenLoops library for the extensive correspondence and for kindly providing us with a private version of OpENLOOPS2. We also thank M. Pellen for a cross-check with the results of Ref. [30]. A. M. thanks the Department of Physics at Princeton University for its hospitality during the completion of this work. The work of A. B. was supported in part by BMBF Project No. 05H18VKCC1 and in part by the DFG Research Grant "Top-Quarks under the LHCs Magnifying Glass: From Process Modelling to Parameter Extraction." The work of M. C. was supported in part by a grant of the BMBF. The research of A.M., A.S.P., and R.P. has 
received funding from the European Research Council (ERC) under the European Union's Horizon 2020 research and innovation programme (Grant Agreement No. 683211). The work of A. M. and A.S.P. was also supported by UK STFC Grants No. ST/L002760/1 and No. ST/K004883/1. A. S. P. is a cross-disciplinary postdoctoral fellow supported by funding from the University of Edinburgh and Medical Research Council (core grant to the MRC Institute of Genetics and Molecular Medicine). Simulations were performed with computing resources granted by RWTH Aachen University under Project No. rwth0313.

[1] V. D. Barger, J. Ohnemus, and R. J. N. Phillips, Spin correlation effects in the hadroproduction and decay of very heavy top quark pairs, Int. J. Mod. Phys. A 04, 617 (1989).

[2] I. I. Y. Bigi, Y. L. Dokshitzer, V. A. Khoze, J. H. Kühn, and P. M. Zerwas, Production and decay properties of ultraheavy quarks, Phys. Lett. B 181, 157 (1986).

[3] W. Bernreuther, A. Brandenburg, and P. Uwer, Transverse polarization of top quark pairs at the Tevatron and the Large Hadron Collider, Phys. Lett. B 368, 153 (1996).

[4] G. Mahlon and S. J. Parke, Angular correlations in top quark pair production and decay at hadron colliders, Phys. Rev. D 53, 4886 (1996).

[5] W. Bernreuther, A. Brandenburg, and Z. G. Si, Next-toleading order QCD corrections to top quark spin correlations at hadron colliders: The reactions $q \bar{q} \rightarrow t \bar{t}(g)$, Phys. Lett. B 483, 99 (2000).

[6] W. Bernreuther, A. Brandenburg, Z. G. Si, and P. Uwer, Next-to-leading order QCD corrections to top quark spin correlations at hadron colliders: The reactions $g g \rightarrow t \bar{t}(g)$ and $g q \bar{q} \rightarrow t \bar{t} q(\bar{q})$, Phys. Lett. B 509, 53 (2001).

[7] W. Bernreuther, A. Brandenburg, Z. G. Si, and P. Uwer, Top Quark Spin Correlations at Hadron Colliders: Predictions at Next-to-Leading Order QCD, Phys. Rev. Lett. 87, 242002 (2001).

[8] G. Mahlon and S. J. Parke, Spin correlation effects in top quark pair production at the LHC, Phys. Rev. D 81, 074024 (2010).

[9] W. Bernreuther and Z. G. Si, Distributions and correlations for top quark pair production and decay at the Tevatron and LHC, Nucl. Phys. B837, 90 (2010).

[10] K. Melnikov and M. Schulze, Top quark spin correlations at the Tevatron and the LHC, Phys. Lett. B 700, 17 (2011).

[11] W. Bernreuther, D. Heisler, and Z. G. Si, A set of top quark spin correlation and polarization observables for the LHC: Standard model predictions and new physics contributions, J. High Energy Phys. 12 (2015) 026.

[12] W. Bernreuther, P. Galler, Z. G. Si, and P. Uwer, Production of heavy Higgs bosons and decay into top quarks at the LHC. II: Top-quark polarization and spin correlation effects, Phys. Rev. D 95, 095012 (2017).

[13] J. A. Aguilar-Saavedra, Dilepton azimuthal correlations in $t \bar{t}$ production, J. High Energy Phys. 09 (2018) 116.

[14] P. Richardson and S. Webster, Spin correlations in parton shower simulations, arXiv:1807.01955.
[15] C. T. Hill and S. J. Parke, Top production: Sensitivity to new physics, Phys. Rev. D 49, 4454 (1994).

[16] Z. Han, A. Katz, D. Krohn, and M. Reece, (Light) stop signs, J. High Energy Phys. 08 (2012) 083.

[17] M. Baumgart and B. Tweedie, A new twist on top quark spin correlations, J. High Energy Phys. 03 (2013) 117.

[18] Z. Han and A. Katz, Stealth stops and spin correlation: A Snowmass white paper, arXiv:1310.0356.

[19] S. Biswas, R. Frederix, E. Gabrielli, and B. Mele, Enhancing the $t \bar{t} H$ signal through top-quark spin polarization effects at the LHC, J. High Energy Phys. 07 (2014) 020.

[20] ATLAS Collaboration, Measurements of top-quark pair spin correlations in the $e \mu$ channel at $\sqrt{s}=13 \mathrm{TeV}$ using $p p$ collisions in the ATLAS detector, CERN Report No. ATLAS-CONF-2018-027, 2018.

[21] S. Chatrchyan et al. (CMS Collaboration), Measurements of $t \bar{t}$ Spin Correlations and Top-Quark Polarization Using Dilepton Final States in $p p$ Collisions at $\sqrt{s}=7 \mathrm{TeV}$, Phys. Rev. Lett. 112, 182001 (2014).

[22] G. Aad et al. (ATLAS Collaboration), Measurements of spin correlation in top-antitop quark events from proton-proton collisions at $\sqrt{s}=7 \mathrm{TeV}$ using the ATLAS detector, Phys. Rev. D 90, 112016 (2014).

[23] V. M. Abazov et al. (D0 Collaboration), Measurement of spin correlation between top and antitop quarks produced in $p \bar{p}$ collisions at $\sqrt{s}=1.96 \mathrm{TeV}$, Phys. Lett. B 757, 199 (2016).

[24] V. Khachatryan et al. (CMS Collaboration), Measurements of $t \bar{t}$ spin correlations and top quark polarization using dilepton final states in $p p$ collisions at $\sqrt{(s)}=8 \mathrm{TeV}$, Phys. Rev. D 93, 052007 (2016).

[25] M. Aaboud et al. (ATLAS Collaboration), Measurements of top quark spin observables in $t \bar{t}$ events using dilepton final states in $\sqrt{s}=8 \mathrm{TeV} p p$ collisions with the ATLAS detector, J. High Energy Phys. 03 (2017) 113.

[26] M. Czakon, P. Fiedler, and A. Mitov, Resolving the Tevatron Top Quark Forward-Backward Asymmetry Puzzle: Fully Differential Next-To-Next-To-Leading-Order Calculation, Phys. Rev. Lett. 115, 052001 (2015).

[27] M. Czakon, D. Heymes, and A. Mitov, High-Precision Differential Predictions for Top-Quark Pairs at the LHC, Phys. Rev. Lett. 116, 082003 (2016).

[28] J. Gao and A. S. Papanastasiou, Top-quark pair-production and decay at high precision, Phys. Rev. D 96, 051501(R) (2017).

[29] A. Denner, S. Dittmaier, S. Kallweit, and S. Pozzorini, NLO QCD corrections to off-shell top-antitop production with leptonic decays at hadron colliders, J. High Energy Phys. 10 (2012) 110.

[30] A. Denner and M. Pellen, NLO electroweak corrections to off-shell top-antitop production with leptonic decays at the LHC, J. High Energy Phys. 08 (2016) 155.

[31] E. L. Berger, J. Gao, C.-P. Yuan, and H. X. Zhu, NNLO QCD corrections to $t$-channel single top-quark production and decay, Phys. Rev. D 94, 071501(R) (2016).

[32] E. L. Berger, J. Gao, and H. X. Zhu, Differential distributions for $t$-channel single top-quark production and decay at next-to-next-to-leading order in QCD, J. High Energy Phys. 11 (2017) 158. 
[33] W. Bernreuther, A. Brandenburg, Z. G. Si, and P. Uwer, Top quark pair production and decay at hadron colliders, Nucl. Phys. B690, 81 (2004).

[34] K. Melnikov and M. Schulze, NLO QCD corrections to top quark pair production and decay at hadron colliders, J. High Energy Phys. 08 (2009) 049.

[35] J. M. Campbell and R. K. Ellis, Top-quark processes at NLO in production and decay, J. Phys. G 42, 015005 (2015).

[36] A. Broggio, A. S. Papanastasiou, and A. Signer, Renormalization-group improved fully differential cross sections for top pair production, J. High Energy Phys. 10 (2014) 098.

[37] M. Czakon, A novel subtraction scheme for double-real radiation at NNLO, Phys. Lett. B 693, 259 (2010).

[38] M. Czakon, Double-real radiation in hadronic top quark pair production as a proof of a certain concept, Nucl. Phys. B849, 250 (2011).

[39] M. Czakon and D. Heymes, Four-dimensional formulation of the sector-improved residue subtraction scheme, Nucl. Phys. B890, 152 (2014).

[40] S. Catani and M. H. Seymour, The dipole formalism for the calculation of QCD jet cross-sections at next-to-leading order, Phys. Lett. B 378, 287 (1996).

[41] S. Catani, S. Dittmaier, M. H. Seymour, and Z. Trocsanyi, The dipole formalism for next-to-leading order QCD calculations with massive partons, Nucl. Phys. B627, 189 (2002).

[42] J. M. Campbell, R. K. Ellis, and F. Tramontano, Single top production and decay at next-to-leading order, Phys. Rev. D 70, 094012 (2004).

[43] M. Czakon, P. Fiedler, D. Heymes, and A. Mitov, NNLO QCD predictions for fully-differential top-quark pair production at the Tevatron, J. High Energy Phys. 05 (2016) 034.

[44] M. Czakon, D. Heymes, and A. Mitov, Dynamical scales for multi-TeV top-pair production at the LHC, J. High Energy Phys. 04 (2017) 071.

[45] F. Buccioni, J. N. Lang, J. M. Lindert, P. Maierhöfer, S. Pozzorini, H. Zhang, and M. Zoller, OpenLoops2, arXiv: 1907.13071.

[46] F. Cascioli, P. Maierhöfer, and S. Pozzorini, Scattering Amplitudes with Open Loops, Phys. Rev. Lett. 108, 111601 (2012).

[47] F. Buccioni, S. Pozzorini, and M. Zoller, On-the-fly reduction of open loops, Eur. Phys. J. C 78, 70 (2018).

[48] L. Chen, M. Czakon, and R. Poncelet, Polarized doublevirtual amplitudes for heavy-quark pair production, J. High Energy Phys. 03 (2018) 085.

[49] P. Bärnreuther, M. Czakon, and P. Fiedler, Virtual amplitudes and threshold behaviour of hadronic top-quark pairproduction cross sections, J. High Energy Phys. 02 (2014) 078.

[50] R. Bonciani and A. Ferroglia, Two-loop QCD corrections to the heavy-to-light quark decay, J. High Energy Phys. 11 (2008) 065.
[51] H. M. Asatrian, C. Greub, and B. D. Pecjak, NNLO corrections to $\bar{B} \rightarrow X(u) l \bar{\nu}$ in the shape-function region, Phys. Rev. D 78, 114028 (2008).

[52] M. Beneke, T. Huber, and X.-Q. Li, Two-loop QCD correction to differential semi-leptonic $b \rightarrow u$ decays in the shape-function region, Nucl. Phys. B811, 77 (2009).

[53] M. A. Lim, Quantum chromodynamics and the precision phenomenology of heavy quarks, arXiv:1811.10874.

[54] G. Cullen et al., GoSAM-2.0: A tool for automated one-loop calculations within the standard model and beyond, Eur. Phys. J. C 74, 3001 (2014).

[55] J. Gao, C. S. Li, and H. X. Zhu, Top Quark Decay at Nextto-Next-to Leading Order in QCD, Phys. Rev. Lett. 110, 042001 (2013).

[56] M. Brucherseifer, F. Caola, and K. Melnikov, $\mathcal{O}\left(\alpha_{s}^{2}\right)$ corrections to fully-differential top quark decays, J. High Energy Phys. 04 (2013) 059.

[57] I. R. Blokland, A. Czarnecki, M. Slusarczyk, and F. Tkachov, Heavy to Light Decays with a Two Loop Accuracy, Phys. Rev. Lett. 93, 062001 (2004).

[58] R. D. Ball et al. (NNPDF Collaboration), Parton distributions from high-precision collider data, Eur. Phys. J. C 77, 663 (2017).

[59] S. Dulat, T.-J. Hou, J. Gao, M. Guzzi, J. Huston, P. Nadolsky, J. Pumplin, C. Schmidt, D. Stump, and C.-P. Yuan, New parton distribution functions from a global analysis of quantum chromodynamics, Phys. Rev. D 93, 033006 (2016).

[60] T. Kluge, K. Rabbertz, and M. Wobisch, FASTNLO: Fast pQCD calculations for PDF fits, arXiv:hep-ph/0609285.

[61] D. Britzger et al. (fastNLO Collaboration), New features in version 2 of the FASTNLO project, arXiv:1208.3641.

[62] M. Czakon, D. Heymes, and A. Mitov, fastNLO tables for NNLO top-quark pair differential distributions, arXiv:1704 .08551 .

[63] A repository with results and additional plots can be found at http://www.precision.hep.phy.cam.ac.uk/results/ttbar-decay/.

[64] M. Cacciari, G. P. Salam, and G. Soyez, The anti- $k_{t}$ jet clustering algorithm, J. High Energy Phys. 04 (2008) 063.

[65] M. Cristinziani and M. Mulders, Top-quark physics at the Large Hadron Collider, J. Phys. G 44, 063001 (2017).

[66] V. Khachatryan et al. (CMS Collaboration), Measurement of differential cross sections for top quark pair production using the lepton + jets final state in proton-proton collisions at $13 \mathrm{TeV}$, Phys. Rev. D 95, 092001 (2017).

[67] M. Aaboud et al. (ATLAS Collaboration), Measurements of top-quark pair differential cross-sections in the lepton + jets channel in $p p$ collisions at $\sqrt{s}=13 \mathrm{TeV}$ using the ATLAS detector, J. High Energy Phys. 11 (2017) 191.

[68] S. Frixione and A. Mitov, Determination of the top quark mass from leptonic observables, J. High Energy Phys. 09 (2014) 012. 